\title{
Mouse splice mutant generation from ENU-treated ES cells-A gene-driven approach
}

\author{
Boris Greber, Hans Lehrach, Heinz Himmelbauer* \\ Department of Vertebrate Genomics, Max-Planck-Institute of Molecular Genetics, Ihnestrasse 73, 14195 Berlin, Germany
}

Received 20 December 2004; accepted 25 January 2005

\begin{abstract}
Mutant mice are important for elucidating mammalian gene functions and for modeling human disease phenotypes. In recent years, chemical mutagenesis has become an increasingly popular method to disrupt gene functions due to its high efficiency of inducing mutations throughout the genome. Mutagenesis of embryonic stem (ES) cells offers the possibility of gene-driven approaches, which, however, require efficient mutation detection procedures to screen archives of mutated samples for lesions in particular genes. We have developed an approach that focuses on the detection of splice mutations in highly pooled cDNA samples using exon-skipping PCR primers. As a proof of concept, splice mutants for the Kit gene were isolated from a library comprising approximately 40,000 ES cell clones treated with $N$-ethyl- $N$ nitrosourea followed by transmission through the mouse germ-line. The approach will be useful for the production of mouse models for human disease-related splice mutations and as a general gene disruption strategy.
\end{abstract}

(C) 2005 Elsevier Inc. All rights reserved.

Keywords: ENU mutagenesis; Reverse genetics; Mutant screening; Splice mutations; Mouse ES cells

Gene-driven screens for mutant production based on chemical mutagenesis have been reported for a range of model organisms, including Arabidopsis [1], Caenorhabditis elegans [2], Drosophila [3], zebrafish [4], rat [5,6], and mouse $[7,8]$. The general approach is to screen large numbers of samples taken from chemically mutagenized individuals for mutations in genes of interest using PCRbased mutation detection strategies such as denaturing HPLC or heteroduplex cleavage by CEL I endonuclease [9]. Upon identification of a mutation, the corresponding individual is recovered and used as a founder for establishing a mutant line. During the screening process, animals may be kept as living stocks, but in the case of the mouse, mutants are preferentially generated from samples of frozen sperm [7] or embryonic stem (ES) cells [8]. Using ES cells as starting material allows for cDNA-based mutation detection enabling the coverage of a gene's coding sequence

\footnotetext{
* Corresponding author. Fax: +49 3084131128.

E-mail address: himmelbauer@molgen.mpg.de (H. Himmelbauer).
}

in a single PCR amplicon. However, mouse production from chemically mutagenized ES cells may be compromised if cells are saturated with mutations throughout the genome $[8,10]$. Choosing accordingly reduced mutagen dosages, i.e., lower mutation frequencies, would require a corresponding increase in library size to ensure the effective isolation of mutations. This in turn would necessitate a screening strategy based on the analysis of pooled samples. However, the sensitivity for the detection of unknown point mutations is limited. For instance, mutation detection via dHPLC or CEL I-mediated heteroduplex cleavage may be applied only to moderately pooled samples in routine operations $[11,12]$. The sensitivity of these established approaches is ultimately restricted by the amount of DNA that can be loaded onto the respective system. With increasing dilutions of mutant against wild type, however, the signal of interest inevitably diminishes due to the increasing background of wild-type PCR fragment. Here we report the feasibility of an alternative strategy that focuses on a another common type of lesion, the detection of exon deletions at the transcript level. Using exon-skipping primers in nested RT-PCR, 
splice mutations can be amplified selectively, concomitantly suppressing the formation of wild-type background.

\section{Results and discussion}

\section{$N$-Ethyl-N-nitrosourea (ENU) mutational spectrum, mutation frequency, and library size}

To obtain information on the ENU mutational spectrum we exposed mouse ES cells to mutagen and subjected surviving cells to negative selection for functional mutations in the X-linked Hprt gene using 6-thioguanine (6-TG) as selective agent. A set of 31 nonredundant clones resistant to 6-thioguanine was characterized at the cDNA level by sequencing of the Hprt open reading frame. As expected, most lesions were point mutations $(23 / 31=74 \%)$. However, a significant percentage of mutations $(6 / 31=19 \%)$ were single-exon deletions, which were most likely caused by point mutations at the genomic level at sites that are relevant for pre-mRNA splicing. This observation is in line with other data [13-15] and opened up the possibility of focusing a screening strategy on this particular type of mutation. Since the exon structure of genes is usually known, deletions of exons at the cDNA level can be detected specifically using exon-skipping primers (Fig. 3D, case 1). To increase the sensitivity of splice mutant detection these PCRs can be performed on PCR amplicons covering the open reading frame of a target gene.

Pooling Hprt deletion clone cDNA against wild-type cDNA followed by nested exon-skipping PCR amplification allowed the identification of splice mutants at ratios up to 1 in 1000 (results not shown), which presents an increase in sensitivity compared with common (point) mutation detection methods of 2 orders of magnitude. We therefore aimed at generating a library of ENU-treated ES cells suitable for the isolation of splice mutant clones. While cell survival after treatment decreased exponentially, mutation frequen- cies based on 6-TG selection (see Materials and methods) increased in a linear manner, with respect to ENU concentration (Fig. 1A). We decided to employ a median mutation rate of 1 in 2500 ( $\approx 10 \%$ of ES cell survival). The probability $(p)$ that a library of given size $(N)$ at a certain mutation frequency (MF) contains $n$ splice mutant clones for an average gene like Hprt (nine exons) may be calculated via the binomial distribution since the number of library clones is small compared to the cell population (a suspension of treated ES cells) it is derived from:

$p=N ! /(N-n) ! / n ! \times \mathrm{MF}^{n} \times(1-\mathrm{MF})^{N-n}$.

Multiplying the desired mutation frequency of $4 \times 10^{-4}$ by $15 \%$ (estimated percentage of exon deletions in the ENU mutational spectrum), the probability of identifying at least one deletion clone $\left(p_{\geq 1}\right)$ equals $1-p_{0} \approx 95 \%$, at a library size of 40,000 clones (Fig. 1B).

\section{Clone library generation}

Hence, we generated a library of approximately this size by pipetting - instead of picking - clones into 96 multititer plates, resulting in a statistical distribution of emerging colonies across the individual positions (chart in Fig. 2). The average number of colonies per well was 4.1 , representing a pooling of samples already at the cellular level. A master copy of the library was frozen for clone recovery while a replica was used for RNA isolation followed by reverse transcription. Facing the high sensitivity of splice mutation detection using nested exon-skipping PCR, cDNA samples were extensively pooled (Fig. 2), resulting in a 24-fold reduction of sample numbers. The average number of clones per pool $(4.1 \times 24 \approx 100=200$ haploid genomes $)$ was nevertheless kept well below the limit of exon deletion detection to account for the possibility of mutant transcript destabilization caused by nonsense-mediated mRNA decay (NMD) [16,17].
A

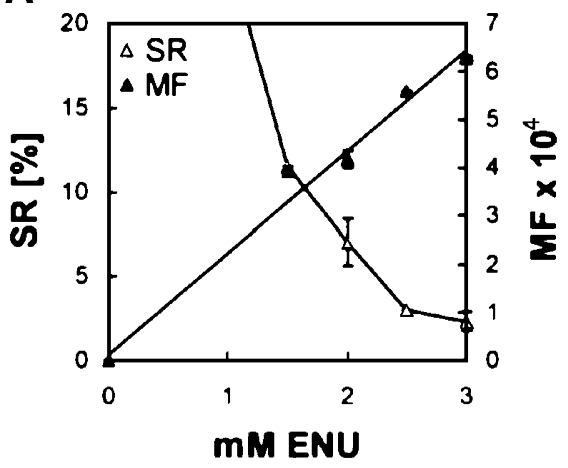

B

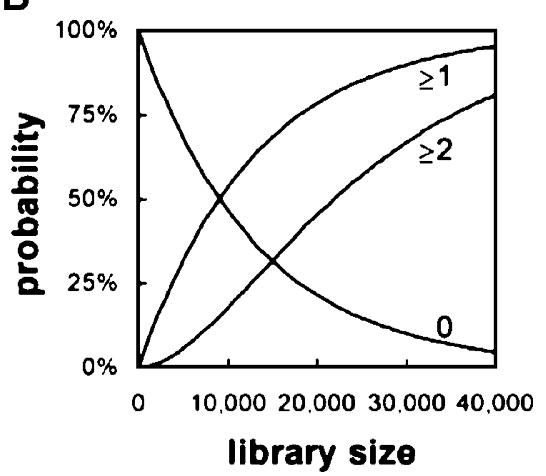

Fig. 1. Relevant aspects regarding clone library generation. (A) ES cell survival and mutation frequencies depending on ENU concentration. Each data point represents one to three independent experiments. Cells were exposed to mutagen for 90 min. SR, survival rate; MF, mutation frequency. Bars indicate standard errors. (B) Theoretical probabilities to detect $0, \geq 1$, or $\geq 2$ splice mutant clones for an autosomal nine-exon gene depending on library size. The mutation frequency was set to $1 / 2500$. Correction factors: two-thirds of the exons accessible by PCR were assumed to be screenable. The percentage of low-quality or lost samples was estimated to be $5 \%$ of the total. 
ENU-treated ES cells

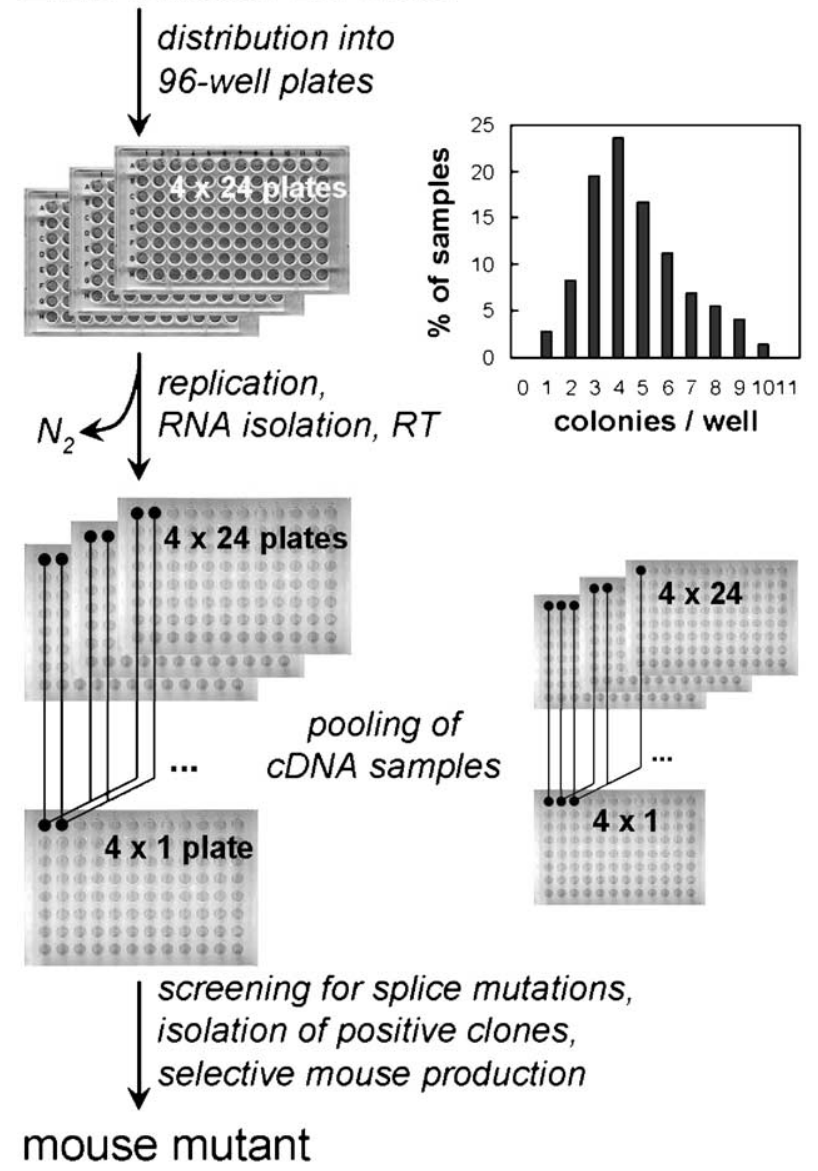

Fig. 2. Flow chart for library generation and sample processing. ENUtreated ES cells were transferred into 9696 -well plates by pipetting, leading to a statistical distribution of emerging colonies across the individual positions (chart on the right). A replica of the library was used for RNA isolation and reverse transcription (RT). cDNA samples were pooled according to two different schemes, enabling the identification of positive plate coordinates and plate numbers by screening pooled samples only. The pools for plate number identification (right) were screened only if positive signals had already been obtained with the samples pooled according to the left scheme. Positive clones could be recovered from the frozen master plates to be employed for mouse mutant generation.

\section{Proof of principle: screen for splice mutations in the Kit} gene

As a proof of concept, we screened the pooled cDNA samples for splice mutations in the Kit oncogene, in which mutations in the $\mathrm{C}$-terminal kinase domain cause a specific dominant phenotype in mice characterized by zones devoid of pigmentation [18]. Care was taken when designing exonskipping primers to prevent artifacts caused by the binding to wild-type template with their $3^{\prime}$ ends (Fig. 3D, case 3 ). Background caused by the nonspecific binding of exonskipping primers to wild-type cDNA (Fig. 3D, case 2) could essentially be eliminated by increasing the annealing temperature. PCRs were analyzed by agarose gel electrophoresis. Fig. 3A shows exon deletion signals obtained for screening Kit exons 6 and 18, representing positive coordinates in the original multiwell plates (Fig. 2, left pooling scheme). In each case rescreening 24 pools generated according to an alternative pooling scheme (Fig. 2 , on the right) allowed the corresponding plate numbers to be identified unequivocally. The respective cell samples were recultivated and several colonies picked for expansion and detection of positive subclones. Positive clones were recovered in both cases as shown in Fig. $3 \mathrm{~B}$ (rescreening by nested exon-skipping PCR). However, if the deletionspecific PCRs were performed as one-step reactions, product yields were significantly reduced compared to the amplification of wild-type Kit cDNA. Since both exon deletions cause frameshifts leading to the generation of premature stop codons more than 50 bp upstream of the $3^{\prime}$-most exon-exon junction, the cause for this observation may be the activity of the NMD [16]. mRNA surveillance can be inactivated by treatment of cells with cycloheximide [19] because NMD activity is dependent on translation. If ES cells were treated with cycloheximide $(100 \mu \mathrm{g} / \mathrm{ml})$ for $4 \mathrm{~h}$ prior to RNA isolation [20], the yields of the deletion-specific PCR products were strongly enhanced for both mutant clones (compare lanes 1 and 3 in Fig. 3C), suggesting a reduction of mutant transcript levels under normal conditions due to NMD action. Nevertheless, the deletion clones had successfully been isolated-despite mRNA surveillance. Cycloheximide pretreatment of library clones does not present a suitable strategy to enhance the amounts of mutant transcripts since levels of spontaneous splice isoforms interfering with exon deletion detection will be elevated, too (results not shown). Hence, the NMD appeared to play an ambivalent role in the screening process: On the one hand, mutant transcripts targeted by NMD are reduced in their amounts, which-in principle - acts against their detection via nested exon-skipping PCR. On the other hand, mRNA surveillance balances erroneous pre-mRNA splicing, which would otherwise lead to the generation of false positive signals. Supporting this view, exons 2, 3, 13, and 17, which do not cause frameshifts when deleted, had to be excluded from the screen due to the occurrence of false positive signals in test reactions using wild-type cDNA as template.

The underlying genomic mutations were investigated in the Kit exon deletion clones by sequencing of genomic PCR products. In both cases, point mutations disrupting the canonical splice donor sites of introns 6 and 18, respectively, were found to be the cause of the exon deletions in the mutant transcripts ( $\Delta$ exon6, GT to GG; $\Delta$ exon18, GT to AT; Fig. $3 \mathrm{E})$, suggesting that the splice mutations were constitutive ones. Screening results for an X-linked gene, however, suggested that also more subtle splice mutation clones may be isolated using nested exon-skipping PCR as a screening procedure (results not shown). Both Kit mutant clones, $\Delta$ exon6 and $\Delta$ exon 18 , were karyotypically normal as judged from chromosome spreads (not shown) and were therefore suitable for chimera production via blastocyst injection. We obtained germ-line transmission for the exon 18 deletion clone. Feet and tail tip of the heterozygous founder were 
A

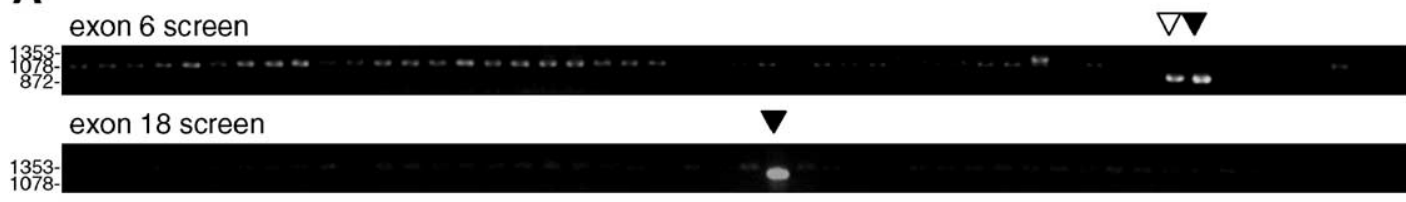

B

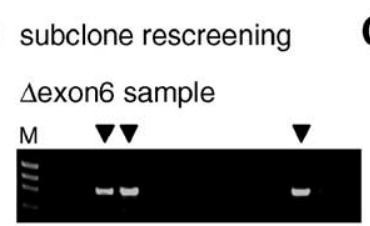

$\Delta$ exon18 sample

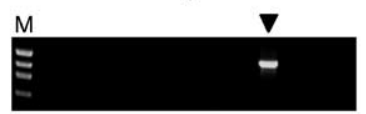

$\mathbf{E}$

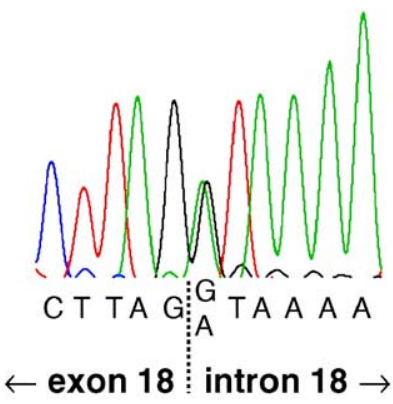

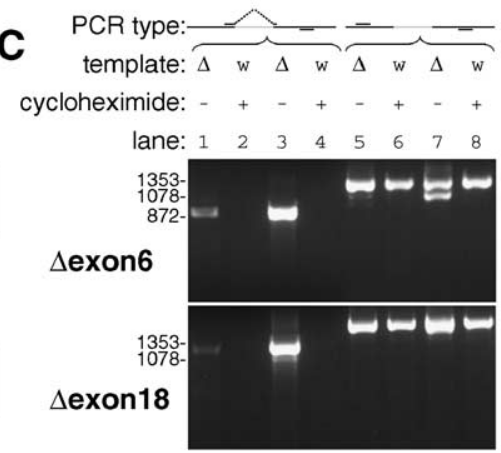

D

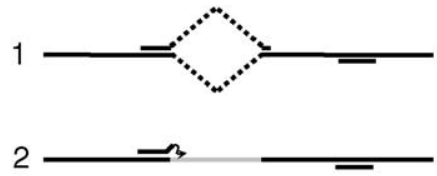

3

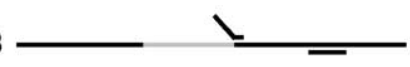

$\mathbf{F}$

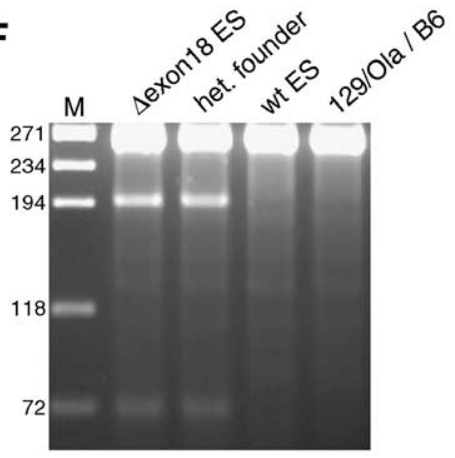

G

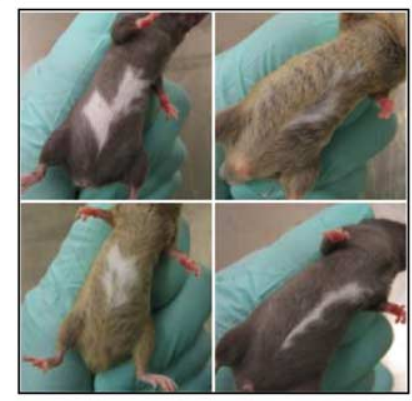

Fig. 3. Screen for splice mutations in the Kit gene. (A) Identification of exon 6 and exon 18 deletion clones in pooled samples. Each gel shows signals for 48 of 384 pooled cDNA samples screened by nested exon-skipping RT-PCR. Sizes of the specific products were 909 and 1201 bp, respectively. Marker band sizes in base pairs are indicated on the left. The band highlighted by the open triangle was not reproducible. (B) Rescreening of subclones established from the positive $\Delta$ exon6 and $\Delta$ exon 18 samples, which consisted of pooled ENU-treated ES cell clones. PCRs were the same as in (A). Lanes representing positive subclones are marked by triangles. (C) Effect of cycloheximide pretreatment on mutant transcript levels. Top: $\Delta$ exon6 ES cell clone. Bottom: $\Delta$ exon18 clone. Lanes 1-4, onestep exon-skipping PCR; lanes 5-8, PCRs across the deletion regions. $\Delta$, deletion clone cDNA as RT-PCR template; w, wild type. cDNA was prepared from ES cells with or without cycloheximide pretreatment as indicated. In lane 7, the $\Delta$ exon18 band was not separated clearly from the wild-type signal during electrophoresis. (D) Possible PCR products associated with exon-skipping primers. Case 1, specific product; case 2, wild-type background formation; case 3, generation of false positive signals. (E) Genomic sequencing of the $\Delta$ exon 18 ES cell clone. In the mutant allele, the intron $185^{\prime}$ splice donor was disrupted by a $\mathrm{G} \rightarrow$ A transition at position +1 . (F) DNA-based genotyping of heterozygous Kit $\Delta$ exon18 mutant mice using the CEL I endonuclease assay. A genomic 270 bp fragment containing the lesion site at position 72 was amplified from $\Delta$ exon18 ES cell DNA, tail-tip DNA of a heterozygous founder animal, wild-type ES cell DNA, and a 1:1 mixture of ES cell (strain 129/Ola) and C57BL/6 DNA. After treatment of the heat-denatured and reannealed PCR fragments with CEL I enzyme the samples were analyzed by agarose gel electrophoresis. In the presence of the mutation, the heteroduplexed PCR product is cleaved into 71.5 - and 198.5-bp subfragments. (G) Germ-line transmission of the Kit $\Delta$ exon18 mutation. The picture shows four of a founder animal's offspring displaying the characteristic dominant belly-spot phenotype.

devoid of pigmentation. To assess if the mutation had been transmitted to this individual, genotyping employing the CEL I endonuclease assay was performed, which we propose as a generally applicable and convenient genotyping strategy. CEL I digests of heteroduplexed PCR fragments across intron 18 yielded products of the predicted sizes for mutated ES cell DNA and DNA isolated from the founder, but not for control samples (Fig. 3F). Further, we obtained offspring from the founder, approximately $50 \%$ of which displayed pigmentation defects, including a white belly spot (Fig. 3G), as is known to be the case for heterozygous Kit mutations that affect the kinase function of the encoded receptor
$[18,21]$. In line with this, the deletion of exon 18 together with the associated frameshift erases a significant portion of the Kit protein's second kinase domain [22]. The function of the Kit protein may be further compromised in our mutant by NMD-mediated destabilization of the altered transcript as shown at the ES cell level (Fig. 3C).

\section{Concluding remarks}

The results presented demonstrate the feasibility of isolating splice mutant clones from an archive of mutagen- 
ized ES cells via screening a relatively low number of highly pooled cDNA samples. This is made possible by the high sensitivity of nested exon-skipping RT-PCR. The analysis of reactions by conventional agarose gel electrophoresis allows for screening samples in a multiparallel way, without need for any sophisticated laboratory equipment. In addition to combining cDNA samples, mutagenized ES cell clones were also pooled during library construction, circumventing the laborious picking of individual colonies. These features make the overall approach particularly time and cost efficient. An increased library size or a moderately enhanced mutation frequency may, however, be required for the isolation of increased numbers of mutated clones per gene.

Splice mutations constitute a particularly interesting type of mutation due to their relevance to human disease $[23,24]$. An earlier study estimated splice mutations to account for approximately $15 \%$ of all disease-causing lesions in humans [25], highlighting the need for efficient approaches to producing mouse splice mutants as models.

\section{Materials and methods}

Mutagenesis, survival rates, mutation frequencies, and mutational spectrum

ES cells of line E14.1 (129/Ola) were grown according to standard procedures [26]. Cells were mutagenized by treatment with ENU for $90 \mathrm{~min}$ at $37^{\circ} \mathrm{C}$ in suspension. To determine survival rates, aliquots of treated ES cells were cultivated for 3 days on dishes to be quantified as colonies under a microscope while correcting the values for plating efficiency. Hprt-based mutation frequencies were measured essentially as described [27]. Briefly, cells surviving the ENU exposure were cultivated for 10 days to allow for HPRT protein depletion in clones harboring functional mutations in the corresponding gene. Then, a subset of the growing cells was replated onto gelatin-coated dishes to be subjected to negative selection with 6-thioguanine $(10 \mu \mathrm{M})$. Resistant colonies were scored after a further 10 days of selection. Mutation frequency was defined as the number of resistant colonies divided by the number of colonies under selection. For characterization of the mutations induced by ENU, RNA was isolated from expanded clones and subjected to reverse transcription. Hprt was amplified employing primers CTTACCTCACTGCTTTCCGG and CTGGCAACATCAACAGGACTC to sequence RT-PCR products subsequently using standard dye-termination technology.

\section{Clone library generation and screening}

The clone library was established from frozen stocks of ES cells treated with $1.5 \mathrm{mM}$ ENU for $90 \mathrm{~min}$. Thawed cells were pipetted into 96 feeder-coated 96-well plates resulting in an average of 4.1 colonies per well. After 1 week of cultivation, aliquots were transferred into new plates and the original ones frozen. Upon the replicas reaching confluency, the ES cells were harvested to serve as starting material for RNA isolation. RNA was isolated using the Trizol reagent (Invitrogen), adapting the manufacturer's instructions to the 96-well format. The modified protocol is available upon request. The average yield of total RNA per sample was approximately $2 \mu \mathrm{g}$. Reverse transcription was performed using reverse transcriptase from Moloney murine leukemia virus (Amersham) and oligo(dT) priming following the company's protocol. One microgram of total RNA was used per $50-\mu 1$ reaction. Aliquots of cDNA from 24 plates each were pooled according to Fig. 2, reducing the total number of 96-well plates to 4 .

Twenty-five-microliter PCRs for the amplification of Kit cDNA contained $65 \mathrm{mM}$ Tris-Cl, $\mathrm{pH} 8.8,16.6 \mathrm{mM}$ $\left(\mathrm{NH}_{4}\right)_{2} \mathrm{SO}_{4}, 0.01 \%$ Tween $20,1-1.5 \mathrm{mM} \mathrm{MgCl}, 0.8 \mathrm{mM}$ dNTPs, $1 \mu \mathrm{M}$ primers, $5 \mathrm{U}$ of a mixture of homemade Taq and $P f u$ polymerases, and cDNA template corresponding to $50 \mathrm{ng}$ of total RNA ( $0.5 \mathrm{ng}$ of RNA equivalent per ES cell clone employing pooled cDNA samples). PCRs were performed using a touchdown protocol and hot-start conditions. As an exception to the rule, the target cDNA was divided into two parts for first-round amplification using primer pairs CTGCTCTGCGTCCTGTTGGTC/GTGGGCTCCGGGAATCCCTCTG (first half) and CGTACGACAGGCTCATAAATGGC/CACGGAATGGTCCACCACCAC (second half). Exon-exon boundaries were determined via the Blat tool at http://genome.ucsc.edu/ using the Kit cDNA sequence as input (NM_021099). Primers used for the nested exon-skipping PCRs were CCTGCTCCGTGGCCAGACAG/CAGGCGAAGTTGGTTCACATAT_CTAC for exon 6 (909 bp, reverse primer skips exon) and CAGAGGGATTCCCGGAGCCCAC/CCCTGGGTAGGGGCTGCTTC_ATTTC for exon $18(1201 \mathrm{bp}$, reverse primer skips exon). The addition of $P f u$ polymerase had to be omitted in the exon-skipping reactions due to its $3^{\prime}$ exonuclease activity. PCRs were analyzed by agarose gel electrophoresis and ethidium bromide staining using large homemade gel chambers with capacity for 384 samples.

\section{Mouse production and genotyping}

Recipient C57BL/6 (B6) embryos were isolated as morulae from superovulated females on day $2.5 \mathrm{pc}$ and cultivated in vitro to the blastocyst stage using M16 medium (Sigma). Kit mutant ES cell clones, $\Delta$ exon6 and $\Delta$ exon18, were injected into blastocysts according to standard procedures [26] followed by embryo transfer preferentially into oviducts of pseudopregnant NMRI females. Highcontribution male chimeras were mated to B6 females.

For mouse genotyping, PCRs were performed using genomic DNA as template and primers CAGCTGCGTGTACACATTTGAA/TTACATTTCGGCAGGCGCG to amplify a 270-bp fragment spanning intron 18 . Fragments were heat denatured and slowly reannealed to allow for hetero- 
duplex formation. Digestion with CEL I endonuclease (Transgenomic) was carried out according to the manufacturer's recommendations. Cleavage products were analyzed by gel electrophoresis $(4.5 \%$ agarose).

\section{Acknowledgment}

This work was supported by the German National Genome Research Network.

\section{References}

[1] C.M. McCallum, L. Comai, E.A. Greene, S. Henikoff, Targeted screening for induced mutations, Nat. Biotechnol. 18 (2000) $455-457$.

[2] G. Jansen, E. Hazendonk, K.L. Thijssen, R.H. Plasterk, Reverse genetics by chemical mutagenesis in Caenorhabditis elegans, Nat. Genet. 17 (1997) 119-121.

[3] A. Bentley, B. MacLennan, J. Calvo, C.R. Dearolf, Targeted recovery of mutations in Drosophila, Genetics 156 (2000) 1169-1173.

[4] E. Wienholds, F. Van Eeden, M. Kosters, J. Mudde, R.H. Plasterk, E. Cuppen, Efficient target-selected mutagenesis in zebrafish, Genome Res. 13 (2003) 2700-2707.

[5] Y. Zan, J.D. Haag, K.S. Chen, L.A. Shepel, D. Wigington, Y.R. Wang, R. Hu, C.C. Lopez-Guajardo, H.L. Brose, K.I. Porter, R.A. Leonard, A.A. Hitt, S.L. Schommer, A.F. Elegbede, M.N. Gould, Production of knockout rats using ENU mutagenesis and a yeast-based screening assay, Nat. Biotechnol. 21 (2003) 645-651.

[6] B.M. Smits, J. Mudde, R.H. Plasterk, E. Cuppen, Target-selected mutagenesis of the rat, Genomics 83 (2004) 332-334.

[7] E.L. Coghill, A. Hugill, N. Parkinson, C. Davison, P. Glenister, S. Clements, J. Hunter, R.D. Cox, S.D. Brown, A gene-driven approach to the identification of ENU mutants in the mouse, Nat. Genet. 30 (2002) 255-256

[8] J.L. Vivian, Y. Chen, D. Yee, E. Schneider, T. Magnuson, An allelic series of mutations in Smad2 and Smad4 identified in a genotypebased screen of $N$-ethyl- $N$-nitrosourea-mutagenized mouse embryonic stem cells, Proc. Natl. Acad. Sci. USA 99 (2002) 15542-15547.

[9] C.A. Oleykowski, C.R. Bronson Mullins, A.K. Godwin, A.T. Yeung, Mutation detection using a novel plant endonuclease, Nucleic Acids Res. 26 (1998) 4597-4602.

[10] R.J. Munroe, R.A. Bergstrom, Q.Y. Zheng, B. Libby, R. Smith, S.W. John, K.J. Schimenti, V.L. Browning, J.C. Schimenti, Mouse mutants from chemically mutagenized embryonic stem cells, Nat. Genet. 24 (2000) 318-321.

[11] M.M. Quwailid, A. Hugill, N. Dear, L. Vizor, S. Wells, E. Horner, S. Fuller, J. Weedon, H. McMath, P. Woodman, D. Edwards, D. Campbell, S. Rodger, J. Carey, A. Roberts, P. Glenister, Z. Lalanne, N. Parkinson, E.L. Coghill, R. McKeone, S. Cox, J. Willan, A. Greenfield, D. Keays, S. Brady, N. Spurr, I. Gray, J. Hunter, S.D.
Brown, R.D. Cox, A gene-driven ENU-based approach to generating an allelic series in any gene, Mamm. Genome 15 (2004) $585-591$

[12] T. Colbert, B.J. Till, R. Tompa, S. Reynolds, M.N. Steine, A.T. Yeung, C.M. McCallum, L. Comai, S. Henikoff, High-throughput screening for induced point mutations, Plant Physiol. 126 (2001) $480-484$

[13] Y. Chen, D. Yee, K. Dains, A. Chatterjee, J. Cavalcoli, E. Schneider, J. Om, R.P. Woychik, T. Magnuson, Genotype-based screen for ENUinduced mutations in mouse embryonic stem cells, Nat. Genet. 24 (2000) 314-317.

[14] M.J. Justice, J.K. Noveroske, J.S. Weber, B. Zheng, A. Bradley, Mouse ENU mutagenesis, Hum. Mol. Genet. 8 (1999) 1955-1963.

[15] J.K. Noveroske, J.S. Weber, M.J. Justice, The mutagenic action of $N$-ethyl- $N$-nitrosourea in the mouse, Mamm. Genome 11 (2000) $478-483$.

[16] L.E. Maquat, Nonsense-mediated mRNA decay: splicing, translation and mRNP dynamics, Nat. Rev. Mol. Cell Biol. 5 (2004) 89-99.

[17] K.E. Baker, R. Parker, Nonsense-mediated mRNA decay: terminating erroneous gene expression, Curr. Opin. Cell Biol. 16 (2004) $293-299$.

[18] P. Besmer, K. Manova, R. Duttlinger, E.J. Huang, A. Packer, C. Gyssler, R.F. Bachvarova, The kit-ligand (steel factor) and its receptor c-kit/W: pleiotropic roles in gametogenesis and melanogenesis, Dev. Suppl. (1993) 125-137.

[19] M.S. Carter, J. Doskow, P. Morris, S. Li, R.P. Nhim, S. Sandstedt, M.F. Wilkinson, A regulatory mechanism that detects premature nonsense codons in T-cell receptor transcripts in vivo is reversed by protein synthesis inhibitors in vitro, J. Biol. Chem. 270 (1995) $28995-29003$.

[20] J.F. Bateman, S. Freddi, S.R. Lamande, P. Byers, S. Nasioulas, J. Douglas, R. Otway, M. Kohonen-Corish, E. Edkins, S. Forrest, Reliable and sensitive detection of premature termination mutations using a protein truncation test designed to overcome problems of nonsense-mediated mRNA instability, Hum. Mutat. 13 (1999) $311-317$.

[21] F. Bernex, P. De Sepulveda, C. Kress, C. Elbaz, C. Delouis, J.J. Panthier, Spatial and temporal patterns of c-kit-expressing cells in WlacZ/+ and WlacZ/WlacZ mouse embryos, Development 122 (1996) 3023-3033.

[22] D. Linnekin, Early signaling pathways activated by c-Kit in hematopoietic cells, Int. J. Biochem. Cell Biol. 31 (1999) 1053-1074.

[23] M. Nissim-Rafinia, B. Kerem, Splicing regulation as a potential genetic modifier, Trends Genet. 18 (2002) 123-127.

[24] N.A. Faustino, T.A. Cooper, Pre-mRNA splicing and human disease, Genes Dev. 17 (2003) 419-437.

[25] M. Krawczak, J. Reiss, D.N. Cooper, The mutational spectrum of single base-pair substitutions in mRNA splice junctions of human genes: causes and consequences, Hum. Genet. 90 (1992) 41-54.

[26] A. Nagy, M. Gertsenstein, K. Vintersten, R. Behringer, Manipulating the Mouse Embryo: A Laboratory Manual, Cold Spring Harbor Laboratory Press, Cold Spring Harbor, NY, 2003.

[27] B. Greber, H. Lehrach, H. Himmelbauer, Characterization of trimethylpsoralen as a mutagen for mouse embryonic stem cells, Mutat. Res. 525 (2003) 67-76. 